Cahiers d'études italiennes

$5 \mid 2006$

NOVECENTO... E DINTORNI

Images littéraires de la société contemporaine (2)

\title{
La seduzione postmoderna in Rimini di Pier Vittorio Tondelli
}

Sabina Gola

\section{OpenEdition}

\section{Journals}

Edizione digitale

URL: http://journals.openedition.org/cei/839

DOI: $10.4000 /$ cei.839

ISSN: 2260-779X

\section{Editore}

UGA Éditions/Université Grenoble Alpes

\section{Edizione cartacea}

Data di pubblicazione: 15 septembre 2006

Paginazione: $219-230$

ISBN: 978-2-84310-086-4

ISSN: 1770-9571

Notizia bibliografica digitale

Sabina Gola, «La seduzione postmoderna in Rimini di Pier Vittorio Tondelli», Cahiers d'études italiennes [Online], 5 | 2006, online dal 15 mars 2008, consultato il 27 mars 2021. URL: http://

journals.openedition.org/cei/839; DOI: https://doi.org/10.4000/cei.839 


\title{
LA SEDUZIONE POSTMODERNA IN RIMINI DI PIER VITTORIO TONDELLI
}

\author{
Sabina Gola \\ Université Libre de Bruxelles (ULB)
}

La famosa cittadina della riviera romagnola che funge da cornice al romanzo di Tondelli ${ }^{1}$ è rappresentata dall'autore come il luogo per eccellenza della seduzione di massa. ${ }^{2}$ Essa viene descritta dall'autore come un immenso parco di divertimenti nel quale vigono regole "altre", nei gesti, nel linguaggio e nei comportamenti. Per i turisti andare a Rimini vuol dire uscire temporaneamente dal rito monotono della quotidianità ed entrare nel mito.

Luogo privilegiato del cammuffamento, dell'illusione e dell'irrealtà, Rimini costituisce a tutti gli effetti un perfetto esempio di non-luogo postmoderno nel quale si evidenzia come principale elemento seduttore il carattere carnevalesco - cioè la possibilità di "trasgredire e invertire le norme sociali abitualmente condivise nella vita quotidiana". ${ }^{3}$ Tutto il romanzo poggia quindi sui binomi realtà/illusione, vero/falso, sostanza/apparenza, interiorità/esteriorità.

Il tentativo di condanna di Rimini nelle pagine conclusive del romanzo, per bocca di un professore di latino, arrivato a Rimini per annunciare l'apocalisse, suona del tutto inutile e non fa che esaltare il carattere consumistico e illusorio della città. Ancora una volta si impone, infatti, la logica del consumo: da un lato, i venditori di sogni, cioè coloro

1. Su Tondelli e la sua opera, consigliamo la lettura di Carnero R., Lo spazio emozionale. Guida alla lettura di Pier Vittorio Tondelli, Novara, Interlinea srl edizioni, 1998.

2. Rimandiamo al commento di Carlo (l'amico di Susy) sulla città romagnola, in Tondelli P.V., Rimini. Corriere della Sera - I grandi romanzi italiani, p. 51. Per le successive citazioni da quest'opera si indicherà d'ora in poi solo il numero di pagina.

3. Righi A., Tondelli: Welcome to Seaside. Le implicazioni del non-luogo come oggetto di narrazione primario in Rimini (1985). http://www.sguardomobile.it/article.php3?id_article=14 
che capiscono che la fine del mondo può avere risvolti economici positivi, e dall'altro, i consumatori di sogni che non pensano che ad appagarli almeno un'ultima volta.

In questo paese delle meraviglie dove tutto è costruito all'insegna del divertimento, della ricerca del piacere e del sogno ad occhi aperti, l'esteriorità diviene specchio dell'anima. Di conseguenza, il corpo ${ }^{4}$ sia nella sua fisicità che negli elementi che servono a "distinguerlo" (abbigliamento, trucco, ... ) e a renderlo quindi più seducente, assume il ruolo fondamentale di rappresentante privilegiato dell'interiorità. La descrizione che il narratore fa della camminata del giovane Aelred calza perfettamente:

Il ragazzo camminava in simbiosi con la propria andatura così naturalmente sovrapposto all'artificialità del suo passo, così completamente abbandonato ai gesti appresi (che parlavano di palestre, di basket ball [sic], di cavalli, di lavoro a tavolino) che il suo carattere si diffondeva, completamente svelato all'esterno. Bruno notò che la sua corazza gestuale non appariva come una difesa, non nascondeva non occultava, anzi parlava chiaramente e dolcemente. La sua camminata, infatti, nient'altro era che il tic del suo animo. (p. 191)

Attraverso il corpo si stabiliscono inoltre rapporti di superiorità e di inferiorità: l'influenza dell'aspetto esteriore sulla realtà è così profonda che l'essere belli fisicamente può portare al successo e quindi all'acquisizione del potere; l'essere brutti all'insuccesso. Da qui il culto per la bellezza e per l'eterna giovinezza. ${ }^{5}$

I personaggi di Rimini si muovono quindi in una società in cui il mondo interiore si manifesta attraverso ciò che si vede. Marco Bauer, protagonista di una delle storie del romanzo, che racconta la sua vicenda in prima persona, guarda i personaggi, con cui interagisce e, in maniera ossessiva, ce ne descrive l'aspetto fisico e l'abbigliamento, mostrandoci quindi solo ciò che i personaggi vogliono farci vedere.

In definitiva, i vari personaggi messi a fuoco da Tondelli ("sotto l'occhio dei riflettori”, p. 52) si muovono come comparse sul palcoscenico di questa grande fabbrica di illusioni che è Rimini, private completamente

4. Sul tema del corpo nella narrativa tondelliana, si veda Buia E., Verso casa. Viaggio nella narrativa di Pier Vittorio Tondelli, Ravenna, Fernandel, 1999. Ne citiamo solo un breve passaggio : "La poetica tondelliana dell'emotività, oltre a permettere una perenne oscillazione tra degrado e esaltazione, investe il corpo di una dirompente carica sensuale, questa ben lontana dall'essere vissuta in maniera tiepida e discreta, dilaga con esuberanza e vivacità, divenendo una componente irrinunciabile in una scrittura che intende calarsi profondamente nelle pieghe della vita. Il rifiuto e la lode della corporeità finiscono per esacerbarsi nella complicanza del discorso amoroso" (p. 21).

5. Marco Bauer invidia la forma fisica e il bel corpo atletico di Guglielmo, amico di Susy. (Rimini, p. 90-91). 
della loro interiorità. Infatti, se da un lato la volontà li spinge ad autodeterminarsi rispetto al mondo circostante e quindi a portare a compimento i propri desideri (modello della quest), dall'altro, sono ignari del fatto che nel non-luogo postmoderno "la soggettività viene eterodiretta, e il dispositivo spettacolare incanala le affermazioni soggettive in soluzioni che però sono anche preordinate e finalizzate secondo silenziosi e rigidi schemi socializzanti". ${ }^{6}$ Lo stesso Tondelli, in un'intervista a Fulvio Panzeri, affermava che "solo poche persone hanno capito che Rimini è un libro di sconfitti". ${ }^{7}$

Molteplici sono i personaggi che affollano il romanzo, ma Tondelli punta i riflettori su alcuni di essi e sulle loro vicende, anch'esse molto diverse sia nel contenuto che nello spazio narrativo: ${ }^{8}$ Marco Bauer, rampante giornalista inviato a Rimini dalla redazione milanese del suo giornale per dirigere il supplemento estivo; Bruno May, giovane scrittore omosessuale; Alberto, musicista notturno che si innamora di Milvia, giovane madre di famiglia in vacanza a Rimini; Beatrix, antiquaria tedesca alla ricerca della sorella scomparsa; Roby e Tony, registi e produttori di un film ambientato sulla riviera; Renato, figlio di una famiglia decaduta di imprenditori romagnoli; un professore di latino arrivato a Rimini per annunciare l'apocalisse. Marco Bauer, Bruno, Alberto e Beatrix sono coinvolti in storie di seduzione, tutte molto diverse e nelle quali la seduzione assume rilevanza e significati diversi.

Alberto e Milvia - donna non bella, per niente appariscente, sempre spettinata, perfetto opposto della donna fatale - vivono la loro breve ma intensa storia d'amore in una camera d'albergo. La loro storia è magistralmente costruita da Tondelli sull'opposizione reale e metaforica di buio e luce. Mentre Milvia rappresenta la luce per Alberto che è prigioniero della notte, Alberto rappresenta per Milvia, presa nell'accecante luce della vita, il misterioso e fantomatico sconosciuto della notte. "E così facendo entravano nel mito: Alberto non era solo un uomo, ma tutti gli uomini di questa terra; e lei, Milvia, tutta la dolcezza recettiva e femminea di questo mondo" (p. 163). Entrambi, scontenti di sé stessi e della loro vita, si seducono reciprocamente per sfuggire all'apatia e alla tristezza della quotidianità. ${ }^{\text {? }}$

6. Righi A., art. cit.

7. Panzeri F., Picone G., Tondelli il mestiere di scrittore, Milano, Bompiani, 2001, p. 58.

8. Nelle parole dello stesso Tondelli, Rimini è il tentativo di descrivere la riviera adriatica "come "contenitore" di storie diverse ... un affresco, forse una sinfonia, della realtà italiana di questi anni e dei vari modi - quello sentimentale, quello drammatico, quello esistenziale - di raccontarla". Si veda Panzeri F., Picone G., op. cit., p. 109.

9. "I due amanti procedevano nei loro abbracci costruendosi reciprocamente una sorta di loro personalissima leggenda" (p. 163). 
Nella storia di Mario e Beatrix, la seduzione si lega strettamente alla ricerca dell'identità e all'amore. Sulla spiaggia di Rimini, Beatrix conosce Mario, che per guadagnarsi un po' di soldi dà lezioni di italiano ai turisti tedeschi. Beatrix, che ormai comincia a disperare di ritrovare Claudia, rimane subito colpita dallo sguardo del giovane professore: "E qual era il sole che l'aveva illuminata? Il sole della costa, il sole dell'Italia o lo sguardo di quel ragazzo, Mario ?" (p. 179). Tondelli non sfugge alla tentazione dello stereotipo. Gli ingredienti ci sono tutti: spiaggia, sole e un affascinante professore di italiano. Mario però non veste minimamente i panni del seduttore, anzi si mostra subito dolce e gentile con Beatrix, assumendo un ruolo fondamentale nella ricerca e nel ritrovamento di Claudia. Beatrix, reduce da un matrimonio fallito e in preda alla disperazione, si lascia sedurre dai modi gentili del giovane, se ne innamora profondamente e gli si concede sessualmente solo quando la sua ricerca (della sorella e, in realtà, anche della propria identità) sarà conclusa.

Il giornalista Marco Bauer si illude di essere stato mandato a Rimini per compiere un salto di qualità nell'ambito della sua professione (è stato incaricato di scoprire la verità su un supposto suicidio). Dalla personalità chiaramente narcisistica, ${ }^{10}$ definito da Peter Brooks "macchina desiderante", ${ }^{11}$ Bauer, arrivato a Rimini, parte alla conquista del suo momento di gloria giornalistica e contemporaneamente dell'oggetto amoroso, incarnato dalla bella Susy, la collega che lo affianca nella redazione del giornale. Se, nell'irrealtà riminese, Bauer appare come l'infallibile scopritore della verità e l'abile seduttore (e Susy la ragazza sedotta), nella realtà, il giornalista è soltanto una pedina della ragazza, incaricata di tenerlo sotto controllo proprio da coloro che si nascondono dietro tutta la faccenda del finto suicidio. In ogni caso, l'esperienza riminese di Bauer si rivela un tragico salto nel vuoto dell'illusione e della sua personalità.

Marco Bauer e Susy sono i protagonisti della storia principale del romanzo: entrambi giornalisti desiderosi di sfondare nella loro professione, senza esclusione di colpi. Tondelli costruisce due personaggi molto simili, ma nello stesso tempo anche molto diversi. Bauer presuntuoso, con tendenza cronica a sopravvalutarsi; freddo e distaccato soprattutto con le donne per evitare di rivelare i propri sentimenti; di gusti mediocri quanto mediocre è la sua preparazione professionale ("lettore da due soldi", per

10. Sul narcisismo che caratterizza la società degli anni Ottanta, e che si incarna nel personaggio Marco Bauer, si veda Krízová K., "Rimini : Personalità e cultura narcisistica"

(http: //www.phil.muni.cz/rom/krizova00.pdf).

11. Righi A., op. cit. 
sua ammissione); incapace di capire gli individui nell'animo, attento osservatore quasi maniacale dell'aspetto esteriore dei personaggi, soprattutto dell'abbigliamento e dei capelli; rigorosamente carente di empatia verso i problemi delle altre persone. Susy è una bella donna, elegante, seducente, arrivista, determinata, sicura di sé, competente nel suo lavoro, abile nella finzione e in grado di capire bene la personalità degli altri (soprattutto di Bauer) e di comportarsi di conseguenza.

Il rapporto che si instaura tra di loro è un rapporto di forza, che troverà soluzione solo alla fine del romanzo, a scapito di Bauer che accecato dall'illusione non è più in grado di interpretare la realtà che lo circonda.

Fin dall'inizio cuore e lavoro si confondono nei pensieri e negli atti di Bauer che non esita a sacrificare il primo in favore del secondo e a sfruttare gli altri per i propri interessi, soprattutto le donne con le quali esce. Da entrambe le relazioni amorose di Bauer, prima con Katy - un'affascinante modella di dieci anni più vecchia di lui che egli non esita a lasciare per impegnare tutte le sue energie nel successo professionale ${ }^{12}$ - poi con Susy, appare chiaramente che per il rampante giornalista i sentimenti hanno ragione di esistere solo se al servizio della carriera e che il sesso, naturalmente slegato dai sentimenti, è solo un divertimento senza impegno.

Il primo incontro-scontro tra Bauer e Susy avviene alla redazione riminese, dopo un primo brevissimo e "gelido" dialogo telefonico la cui funzione principe è quella di mettere in evidenza la voce calda e suadente di Susy attorno alla quale si crea un alone di mistero. Susy arriva alle spalle di Bauer, inaspettamente - il fatto di comparire all'improvviso sembra essere una delle particolarità che la rendono ancora più seducente e misteriosa annunciandosi con la voce. Bauer stesso ce la presenta, descrivendone come del resto fa con gli altri personaggi, l'aspetto fisico e l'abbigliamento:

Mi voltai. Era lì che si stava togliendo la giacchetta gettandola distrattamente sul divano. Era la principessa e che razza di principessa. Aveva capelli neri, occhi neri, pelle abbronzata, un paio di gambe affusolate inguantate in calze trasparenti nere, scarpe col tacco alto anch'esse nere. E una camicetta senza maniche di seta bianca che lasciava indovinare un paio di tette da schianto, ritte e dai grandi capezzoli scuri. Mi chiamo Susanna Borgosanti, disse la fata. Era una favola, una bellissima favola. ${ }^{13}$

12. Nel momento dell'ultimo amplesso con Katy, che egli stesso definisce "masturbazione", Bauer si dice: "anche tu non fai più per me. Come il vecchio bar, come la vecchia stanza di redazione. Siete tutti arredi del mio passato. Io vi sto lasciando e quel che è peggio è che non ho rimorsi. Vi lascio come si lascia una lunga, noiosa convalescenza. Per vivere" (p. 31).

13. P. 37. Il commento venale accompagna sempre la descrizione di Susy (cfr. p. 91). 
Bauer rimane "naturalmente" sedotto da Susy (altre descrizioni lo confermano, cfr. p. 92), senza però rendersene conto. In effetti, in netto contrasto con la sua professione di giornalista, egli non si spingerà mai ad interpretare il loro rapporto, mostrando così la propria cecità, causa principale della sua ingenuità. Egli accontenta l'occhio, la osserva, mettendo in risalto, nelle sue descrizioni, i gesti più sensuali:

Susy rise apertamente. Era la prima volta che lo faceva da quando l'avevo conosciuta, qualche ora prima. Rise spingendo avanti la bocca e aprendo le labbra in modo da spalancare la visione dei denti bianchissimi e serrati. Il suono della risata aveva qualcosa di estremamente infantile. Si arrotolava su se stesso per riprendere poi più squillante. Solamente nel momento in cui portò con eleganza le dita contro le labbra qualche secondo più tardi - parve placarsi. (p. 42)

Sempre attento all'esteriorità, Bauer si lascia sfuggire il significato reale di alcuni atteggiamenti della bella collega nei suoi confronti, come ad esempio la sua apparente sottomissione alle decisioni che lui prende in redazione (“- Sei tu il capo, no?”, p. 44). Accecato dal suo desiderio di successo professionale, Bauer interpreta questa docilità in termini di seduzione, non rendendosi conto di avere di fronte il suo doppio, una donna bella, disinvolta, sicura di sé, caratteristiche che insieme creano un effetto di seduzione irresistibile ma anche la premessa indispensabile per la conquista del potere. "La principessa doveva sempre essere trattata con il velluto. Mi adeguai. La accarezzavo, la gingillavo, la gratificavo, e tutto sommato, la lasciavo in pace. E lei veniva dalla mia parte come attratta da una forza lenta, ma irreversibile" (p. 46).

Se Susy sembra sentirsi a suo agio nell'illusorio universo riminese "l'importante è farlo credere. E crederci" (p.51), risponde serafica all'obiezione di Bauer che Viale Ceccarini a Rimini non è il Sunset Boulevard o la Quinta Strada - Bauer inizialmente si muove con goffaggine. Partito in avanscoperta in un luogo a lui sconosciuto - Bauer non conosce Rimini neppure di fama e non ne conosce quindi le risorse (prima di parlare con alcuni amici di Susy crede che il mare sia l'attrattiva maggiore della riviera) - si scontra con una giovane drogata (Claudia, la sorella di Beatrix) che lo obbliga a farla salire sull'auto. "La ragazza gorgheggiò qualcosa, fece un paio di inchini al suo pubblico e salì come su di una Limousine. E naturalmente l'autista ero io" (p. 47). Questo breve episodio, apparentemente insignificante, ci dà invece indicazioni utili sullo sviluppo della storia. In effetti, Bauer si comporta inconsapevolmente per tutto il romanzo come un "autista"; ${ }^{14}$ il ruolo che Tondelli gli affida è

14. Anche in un'altra occasione Bauer si sente trattato da "semplice autista". Quando riaccompagna a Rimini Bruno May che aveva ritrovato al monastero di Badia Tebalda (p. 145). 
quello di condurre l'opinione pubblica alla conoscenza di una verità apparente ben diversa da quella reale e se stesso alla scoperta della vuotezza della sua personalità.

Mosso da fantasie di illimitato successo, Bauer si sente unico e speciale, l'attore principale su un palcoscenico che è, in realtà, un'intera città. ${ }^{15}$ Forte delle sue velleità professionali, egli tenta la conquista della bella collega, sia sul piano professionale che su quello amoroso, facendo attenzione "da buon giornalista" a ogni dettaglio della personalità della giovane per scoprirne le debolezze e poterle così sfruttare a suo favore. Ma Susy non si lascia prendere nemmeno un momento nella rete di Bauer, malgrado le varie strategie utilizzate. Bauer la provoca nell'orgoglio, poi la lusinga scusandosi. Susy sfodera la sua sensualità. Ha già capito quanto Bauer sia narcisista e per questo, lusingandolo a sua volta, vince il round:

Dischiuse le labbra in un accenno di sorriso. "Questo non mi riguarda, Marco". Era la prima volta che pronunciava il mio nome. Fu un buon colpo, da parte sua. Non ci si abitua mai abbastanza a essere chiamati dagli estranei con il proprio nome di battesimo. È sempre un battito un po' strano e piacevole essere riconosciuti per quelle quattro sillabe e quando ciò accade partendo dalle labbra di una bella donna, bene allora ha un senso quasi magico. Lo si interpreta come una promessa. E si è stupidamente felici di abbassare la guardia. Di cedere l'onore delle armi. Va bene Susy sussurrai, ho capito. (p. 53)

Tra i due si apre una sfida fatta di scontri aperti e di scaramucce che durerà fino alla fine del romanzo e che vedrà Susy vincere su tutta la linea. Più volte, per riprendere in mano la situazione, Bauer cerca di ristabilire le gerarchie professionali ma, in realtà, senza che lui se ne accorga, il gioco lo conduce lei imponendosi anche dal punto di vista professionale. ${ }^{16}$

Susy ha trovato l'io del narcisista seduttore, lo ha snidato e lo ridurrà gradualmente in briciole, senza comunque che Bauer, fino alla fine, se ne renda conto :

Con Susy tutto succedeva come in una estenuante partita a scacchi. Mossa dopo mossa, ora all'attacco, ora in difesa, cercavamo reciprocamente di stanarci. Nessuno dei due aveva in tasca lo schema vincente. Per il momento ci studiavamo, ci guardavamo, ci annusavamo. Ognuno fermo e rigido sulle proprie posizioni. (p. 97)

15. La visione della riviera illuminata dalle insegne e dai fari delle automobili, descritta come un vero e proprio palcoscenico, lo porta a immaginare e ad assaporare già il suo illusorio successo : "E quella strada che per chilometri e chilometri lambiva l'Adriatico offrendo festa, felicità e divertimento, [...] quella stessa scia di piacere segnava il confine tra la vita e il sogno di essa, la frontiera tra l'illusione luccicante del divertimento e il peso opaco della realtà ... tutto questo mi piacque e mi eccitò; forse anche mi confuse..." (p. 52).

16. Durante uno dei servizi che Marco e Susy realizzano insieme, quello sulla "spiaggia dei desideri”, Susy mostra chiaramente la sua autorità professionale e la sua capacità di imporsi su Bauer (cfr. p. 93). 
Dopo un ennesimo scontro "professionale" tra i due e le ennesime scuse di Bauer, il sarcasmo della voce di Susy lo intenerisce e scatena la sua voglia di possedere infine quella che lui crede essere la sua preda. Per non esporsi troppo, dice che lo fa per interpretare il desiderio di entrambi:

Le cinsi la vita con un braccio. La sentii morbida e flessuosa come un'arpa.

"Vieni a dormire da me ... Subito."

Fu sul punto di dire qualcosa, ma non sentii niente. Mi guardò.

"Perché me lo chiedi?"

"Perché è quello che vogliamo tutti e due. Fin dall'inizio."

Fece sì con la testa. (p. 108)

La scena si trasferisce dall'aeroporto all'appartamento di Bauer. Scoppia un temporale e c'è un'interruzione di corrente. Bauer, incerto della sua conquista, non riuscendo più a trovare Susy nel buio della stanza, pensa che lei ne abbia approfittato per andarsene; invece Susy approfitta del buoi per riprendere il controllo della situazione e condurre lei l'atto sessuale. Bauer, naturalmente, non si accorge di nulla. Anzi è talmente orgoglioso della sua conquista ("La vicinanza di Susy mi inorgogliva. Mi sarei congratulato con me stesso", p. 185) che non pensa minimamente che Susy possa usare anche nei suoi confronti l'ambiguità di cui fa uso regolarmente con gli altri ("Riusciva a essere convincente anche nelle situazioni più contraddittorie”, p. 186). Questa sua ambiguità la rende ancora più seducente e desiderabile ai suoi occhi. Pur di suscitare ammirazione negli altri e sentirsi quindi appagato, Bauer ufficializza il loro rapporto, e la chiama "la mia ragazza" quando prende le sue difese contro lo scrittore Bruno May che l'aveva aggredita durante l'intervista.

La morte di Bruno sconvolge il giornalista (Bruno aveva tentato di sedurre Bauer, che lo aveva immediatamente scoraggiato). Bauer, che cerca l'atto sessuale nei momenti di maggior vulnerabilità, telefona a Susy e "la implora" di andare a casa sua. Per la prima volta Bauer si ascolta, sente la sua voce ("una spugna fradicia di alcool e di lacrime", p. 234) e comincia a vacillare. Per un attimo sembra acquisire doti umane, che perderà però subito il giorno dopo quando tenta di ristabilire la sua superiorità professionale su Susy. Susy però non ci sta, si ribella e la sua ribellione scatena le voglie di Bauer che ancora una volta si accorge della sua bellezza e, per la prima volta, del suo potere di seduzione:

Forse davvero quella donna mi stava stregando. Aveva un modo particolare di ricevere i miei abbracci [...]. Ci sapeva fare. Conosceva ormai le mie debolezze in fatto di corpo femminile e se le teneva care. Riusciva a giocarci, a offrirle, per poi subito riprendersele finché non tornavo all'attacco con forza. (p. 239) 
Neanche il paragone dell'organo sessuale di Susy con una pianta carnivora lo rende consapevole del fatto che "sicuramente" quella donna lo stava stregando. Proprio in questa scena di sesso si mette in luce la vera natura del loro rapporto: "Non eravamo due forze opposte, ma la somma di due spinte che tentavano di raggiungere la stessa meta" (p. 240).

Come da copione, alla fine dell'amplesso, Bauer pensa al lavoro, alla sua indagine e improvvisamente ha l'illuminazione decisiva per risolvere il caso. A partire da questo momento, Susy diviene più intima, e Bauer invece non sa cosa dire, non essendo abituato a una relazione seria. Così preferisce pensare solo al lavoro. Si mette qui in evidenza un'altra differenza fondamentale tra i due: mentre in Susy le pulsioni verso l'appagamento sessuale e quelle mirate al successo professionale coesistono, in Bauer sono separate.

La situazione evolve abbastanza velocemente: Bauer capisce di essere stato usato. Ha paura, beve, chiama Susy e le rivela tutto, per scoprire qualche istante dopo di essere stato ingannato anche da lei.

Bauer è un personaggio statico. Il suo comportamento rimane invariato nel corso di tutta la storia: occulta la sua vulnerabilità ed esibisce continuamente la sua "supposta" superiorità. La sua convinzione di essere il migliore condiziona infatti la sua visione delle cose e gli impedisce di comprendere gli avvenimenti e, soprattutto, di decifrare il comportamento degli altri. Egli usa il suo potere di seduzione come strumento per arrivare al successo e uscire da quella situazione di inadeguatezza e di inferiorità iniziale. In realtà Bauer viene sedotto due volte, prima dall'atmosfera incantatrice di Rimini, poi da Susy.

Susy è una donna ambigua e imprevedibile, in continua metamorfosi. Questo la rende rappresentativa della società in cui si muove, una società che richiede ai suoi membri una capacità di adattamento indipendente da ogni senso morale. Con Susy, Tondelli richiama l'archetipo femminile dell'angelo seduttore che però si adegua alle regole della società postmoderna e ai suoi modelli di comportamento.

La sconfitta "amorosa" di Bauer è coerente con il comportamento abituale del personaggio. La sua personalità narcisistica lo induce a pensare di avere ottime doti di seduttore, ma in realtà il suo narcisismo si risolve solo in un vuoto apparire, in un mero sforzo esteriore; il suo fascino è fugace come un fulmine, perché dietro l'apparire si percepisce ben presto una inconsistenza interiore. La sua seduzione o meglio seduttività maschera il nulla. ${ }^{17}$

17. Sulla differenza tra seduzione e seduttività si veda Carotenuto A., Riti e miti della seduzione, Bompiani, 1994. 
Bruno May e Aelred, giovane artista, si conoscono a Londra in occasione dell'inaugurazione di una mostra di sculture. Bruno si sente profondamente attratto da questo giovane dagli occhi "stretti" e "selvaggi". Lo presenta a un critico d'arte e Aelred lo ringrazia invitandolo a mangiare insieme a lui. Improvvisamente però se ne va dal ristorante lasciando Bruno da solo che, risentendosene, decide di tornare a casa. Aelred lo richiama per telefono e propone di recarsi a casa sua. Così inizia la storia di Bruno e Aelred, che si concluderà con la tragica morte di Bruno a Rimini durante la cerimonia per l'assegnazione di un premio letterario.

Fin dal primo sguardo, i due giovani si seducono reciprocamente e in modo fulmineo. Bruno rimane immediatamente sedotto dagli occhi e dalla voce di Aelred ${ }^{18}$ che, a sua volta, rimane colpito dalla bellezza di Bruno. Bruno sta attraversando un momento difficile, è alla ricerca della propria identità e ciò gli impedisce di scrivere. L'incontro con Aelred potrebbe aiutarlo ad uscire dall'impasse esistenziale in cui si trova e quindi gli si concede completamente. Aelred è uno "spostato" (p. 199), secondo i suoi stessi amici che mettono in guardia Bruno dal frequentarlo, uno che "non sa chi è e non potrà mai amare nessuno finché non lo scoprirà. E lo dovrà fare da solo" (p. 202), sono le parole di Padre Anselme, amico di Bruno. Accecato dall'amore, Bruno non vuole ascoltarli, ma ben presto si accorgerà che Aelred è un giovane instabile e un profittatore.

Malgrado la mutevolezza e l'ambiguità di Aelred, entrambi vengono immediatamente travolti dall'amore reciproco. Il loro rapporto non è però ad armi pari (come quello tra Bauer e Susy): Aelred è più debole e quindi Bruno assume un ruolo quasi paterno nei suoi confronti. Dopo il loro primo amplesso, Bruno contempla il corpo del giovane compagno e ringrazia Dio "per avergli fatto conoscere, attraverso il corpo di Aelred, la preghiera nascosta e universale delle sue creature" (p. 197).

Lo stato di abbandono totale non dura molto a causa dell'instabilità di Aelred che non è ancora certo delle sue scelte sessuali. Tra di loro infatti si insinua una donna che per Bruno rappresenta l'"essenza stessa della femminilità" (p. 198).

Il rapporto tra Bruno e Aelred si costruisce sull'abbandono, inteso sia come fusione che come allontanamento, e sulla riconciliazione.

Tornare a Firenze fu per Bruno come tornare a guardarsi nello specchio: riconoscere i tratti del proprio viso, i propri lineamenti, le proprie espressioni. Da troppi mesi

18. "Va bene Aelred, hai vinto. Molte altre volte, innumerevoli altre volte quelle parole sarebbero uscite con lo stesso spasimo di tenerezza e abbandono dal profondo del suo [di Bruno] animo" (p. 194$)$. 
infatti il viso che trovava quando si specchiava non era il suo, ma quello pallido di Aelred.

[...] Bruno provò quella particolare e strana dolcezza che è solo dell'abbandonato [...]: il sentirsi cioè ancora fidanzato per il resto della propria vita, ma fidanzato in assenza. Questo particolare sentimento allora gli si riversava addosso come venerazione di un corpo che l'amato aveva venerato. Attraverso un tale gioco di proiezioni Bruno sentì di amarsi, di voler continuare a esserci, di voler scrivere. Sapeva che nessuno mai al mondo avrebbe potuto togliergli il ricordo del suo amore. Aelred era come incollato alla sua pelle. (p. 209)

L'uno senza l'altro sono perduti, soprattutto Bruno che è sicuro di aver trovato la persona ideale che ha sempre sognato di aver accanto per tutta la vita.

Bruno, sempre stordito dall'alcool, vive nel ricordo ossessivo dell'amante. Al ritorno di Aelred, dopo la prima separazione, alla sua dichiarazione d'amore assoluto e alla sua domanda di perdono, Bruno singhiozzando gli dice: "Poiché tu sei il mio Dio, Aelred, di cosa dovrei perdonarti?" (p. 201).

Alla fusione fisica totale di questi due giovani si aggiunge anche la dimensione spirituale. Proprio a causa di questa dimensione spirituale, il dolore della separazione cresce di volta in volta, soprattutto in Bruno, più cosciente del significato assunto dal loro amore: "Sapeva di uscire da un incubo, ma sapeva altrettanto bene che un altro incubo, molto più terribile, quello dell'abbandono e dell'addio, lo attendeva" (p. 202).

Non solo il rapporto tra uomo e uomo, ma anche quello tra uomo e Dio è sentito come corporeo, solo così può risultare profondo e vitale: un vero e proprio legame d'amore. La finitezza di un gesto, purché d'amore, è in grado di superare la singolarità del momento per schiudere significati oranti e universali. ${ }^{19}$

La storia di Bruno e Aelred mutatis mutandis viene ripresa nel romanzo successivo di Tondelli, Camere separate. ${ }^{20}$ Mentre per Leo la scrittura è un modo per sopravvivere e scappare al vuoto affettivo, per Bruno essa è strumento di morte. Bruno non riesce a scrivere né nell'unione né nella separazione:

Certi giorni cercava di scrivere, di riordinare gli appunti del suo romanzo, ma erano sempre tentativi destinati al fallimento. Non riusciva a entrarci, a mettersi tranquillo, a incatenarsi per il futuro a un'avventura esclusiva e totale come quella della scrittura. (p. 199)

19. Buia E., op. cit., p. 31.

20. Ibid., p. 32. 
Quando si decide a separarsi definitivamente da Aelred - "Dobbiamo separarci. Siamo due deboli attaccati disperatamente uno all'altro. Con una forza sovrumana. Se c'è un mistero nel nostro amore è tutto qui" (p. 221) - e a tornare a Firenze per scrivere, si rende conto che una volta liberatosi dal peso dell'amore si è liberato anche dell'essenza stessa della vita. Non gli rimane che il suicidio per fuggire al suo disagio esistenziale.

Nella storia di Bruno e Aelred, seduzione è lacerazione, segnale di un profondo disagio esistenziale, messo in rilievo proprio dall'incontro tra $\mathrm{i}$ due. La fusione dei corpi e la spiritualità di cui si caricano la corporeità e la sensualità non aiutano Bruno nella ricerca della propria identità, a mettere ordine nel groviglio dei propri sentimenti (neppure Padre Anselme riesce a "guidarlo" nel disordine della sua anima), anzi lo conducono sempre più verso il baratro. Neppure l'abbandono è un approdo. La scissione tra i due "corpi" provoca la morte di una parte di essi e dal dolore della separazione deriva solo angoscia. 